\title{
Autosomal recessive proximal renal tubular acidosis
}

INSERM

\section{Source}

INSERM. (1999). Orphanet: an online rare disease and orphan drug data base. Autosomal recessive proximal renal tubular acidosis. ORPHA:93607

Autosomal recessive proximal renal tubular acidosis (AR pRTA) is a rare form of proximal renal tubular acidosis (pRTA; see this term) characterized by an isolated defect in the proximal tubule leading to the decreased reabsorption of bicarbonate and consequentially to urinary bicarbonate wastage along with additional characteristic clinical features. 